\title{
ЭЛЕКТРОННЫЕ СОСТОЯНИЯ НА ПОВЕРХНОСТИ (100) ИОННЫХ КРИСТАЛЛОВ С УЧЕТОМ СПИН-ОРБИТАЛЬНОГО ВЗАИМОДЕИСТВИЯ
}

Электронная структура на поверхности диэлектриков изучена весьма слабо. Лишь в последнее время появилось несколько экспериментальных работ по изучению поверхностных состояний (ПС) в экситонной $\left[{ }^{1}\right]$ и рентгеновской $\left[{ }^{2}\right]$ областях энергий. Теория ПС в ионных кристаллах развита также недостаточно. Качественные оценки [ $\left.{ }^{3}\right]$, не учитывающие реальную зонную структуру, предсказывают образование пары ПС в запрещенной щели у краев зоны проводимости и валентной зоны. Проведенный нами в $\left[{ }^{4}\right]$ более детальный расчет для кристалла $\mathrm{KCl}$ показывает, что ПС в запрещенной щели появляются только у верхнего края валентной зоны на расстоянии 0,14 э $B$ от нее в центре двумерной зоны Бриллюэна. *

Наиболее богатую и интересную структуру ПС можно ожидать в кристаллах с большой величиной спин-орбитального (СО) взаимодействия для валентных состояний, т. е. в кристаллах с тяжелыми анионами. Заметим, что ожидаемая величина отщепления поверхностных уровней, как правило, меньше, чем величина СО расщепления в центре валентной зоны, поэтому для реальной оценки числа и положения ПС учет СО взаимодействия обязателен практически для всех ионных кристаллов.

В данной работе мы применим технику функций Грина в модели сильной связи для расчета электронной структуры на поверхности (100) в ряду кристаллов - $\mathrm{KCl}, \mathrm{KBr}$ и $\mathrm{KI}$ - с возрастающей величиной $\mathrm{CO}$ связи. Мы покажем, что СО взаимодействие не только существенно влияет на положение ПС в запрещенной щели, но и способствует появлению дополнительных уровней внутри валентной зоны, существование которых было предсказано в [ [ $]$.

\section{1. Влияние СО взаимодействия на валентную зону}

Хотя общий характер расщепления валентной p-зоны щелочногалоидных кристаллов под влиянием СО взаимодействия хорошо известен (см., напр., обзор [ $\left.\left[{ }^{7}\right]\right)$, подробные расчеты различных характеристик, в частности плотности состояний, в литературе отсутствуют. Поэтому мы изложим прежде всего результаты расчетов структуры валентных

* В [5] для кристалла $\mathrm{CsBr}$ на поверхности (110) предсказаны гораздо бо́льшие энергии связи ПС; эта работа содержит, однако, явную ошибку. 
зон изучаемых кристаллов. Как и в $\left[{ }^{8}\right]$, используем метод сильной связи. СО эффекты учитываются в приближении Паули как возмущение, снимающее вырождение $p_{1 / 2}$ и $p_{3 / 2}$ состояний иона галоида. В этих приближениях гамильтониан и волновую функцию кристалла можно представить в виде

$$
\begin{gathered}
H=H_{0}+H_{s 0}, \\
\Psi_{n \mathbf{k}}(\mathbf{r})=\sum_{l, \alpha} C_{l \alpha}(\mathbf{k}, n) \varphi_{\alpha}\left(\mathbf{r}-\mathbf{R}_{l}\right) .
\end{gathered}
$$

Здесь $H_{0}$ - часть гамильтониана, не содержащая членов, связанных с СО взаимодействием, $\mathbf{k}$ - волновой вектор, $n$ - номер электронной (дырочной) зоны, $\varphi_{\alpha}\left(\mathbf{r}-\mathbf{R}_{l}\right)$ - локализованные на узле $\mathbf{R}_{l}$ функции базиса (спиноры) типа $\alpha$, которые считаются далее ортонормированными. В силу теоремы Блоха

$$
C_{l \alpha}(\mathbf{k}, n)=\frac{1}{\sqrt{N}} b_{\alpha}(\mathbf{k}, n) \exp \left(i \mathbf{k} \mathbf{R}_{l}\right) .
$$

Тогда уравнение Шредингера приводится к виду

$$
[H(\mathbf{k})-E(\mathbf{k})] b(\mathbf{k})=0,
$$

причем матрица $H(\mathbf{k})$ имеет структуру

$$
H(\mathbf{k})=\left(\begin{array}{cc}
A & B \\
-B^{*} & A^{*}
\end{array}\right)
$$

где $A^{*}$ означает матрицу, комплексно-сопряженную $A$.

В качестве базиса мы используем $4 s$-функцию $\mathrm{K}^{\circ}$ и три $p$-функции галоида $\left(3 p, 4 p\right.$ и $5 p$ для $\mathrm{Cl}^{-}, \mathrm{Br}^{-}$и $\mathrm{I}^{-}$соответственно), умноженные на спиновые функции. Тогда

$$
b=\left(\begin{array}{l}
b_{1} \\
b_{2}
\end{array}\right) ; \quad b_{1,2}=\left(\begin{array}{l}
b_{s+,-} \\
b_{x+,-} \\
b_{y+,-} \\
b_{z+,-}
\end{array}\right)
$$

Если в гамильтониане $H_{s 0}$ (в представлении, где матрицы операторов $\hat{j}, \hat{m}_{j}$ диагональны) сохранить только диагональные члены, то СО эффекты можно учесть с помощью лишь одного параметра $3 \Delta$, равного величине расщепления состояний $p_{1 / 2}$ и $p_{3 / 2}$ валентной зоны в точке $Г$. При этом

$$
A=\left(\begin{array}{cccc}
H_{00} & H_{01} & H_{02} & H_{03} \\
H_{01}^{*} & H_{11} & H_{12}+\Delta i & H_{13} \\
H_{02}^{*} & H_{12}^{*}-\Delta_{i} & H_{22} & H_{23} \\
H_{03}^{*} & H_{13}^{*} & H_{23}^{*} & H_{33}
\end{array}\right), \quad B=\left(\begin{array}{cccc}
0 & 0 & 0 & 0 \\
0 & 0 & 0 & \Delta \\
0 & 0 & 0 & \Delta i \\
0 & -\Delta & -\Delta i & 0
\end{array}\right),
$$

где $H_{\alpha \beta}$ - матричные элементы гамильтониана $H_{0}$.

Учитывая взаимодействия до вторых соседей включительно и используя свойства симметрии $H_{\alpha \beta}$, эти элементы можно выразить через шесть параметров. Конкретные выражения для $H_{\alpha \beta}$ приведены в $\left[{ }^{8}\right]$. Детерминант системы (4) имеет при этом четыре двукратно вырожденных корня, определяемые секулярным уравнением 


$$
\begin{aligned}
& H^{\prime}{ }_{00} H^{\prime}{ }_{11} H^{\prime}{ }_{22} H^{\prime}{ }_{33}-H^{\prime}{ }_{00} H^{\prime}{ }_{11} H_{23}^{2}-H^{\prime}{ }_{00} H_{22}^{\prime} H_{13}^{2}-H^{\prime}{ }_{00} H^{\prime}{ }_{33} H_{12}^{2}-H^{\prime}{ }_{11} H_{22}^{\prime} H_{03}^{2} \\
& -H^{\prime}{ }_{11} H^{\prime}{ }_{33} H_{02}^{2}-H^{\prime}{ }_{22} H^{\prime}{ }_{33} H_{01}^{2}+2 H^{\prime}{ }_{00} H_{12} H_{13} H_{23}+2 H^{\prime}{ }_{11} H_{02} H_{03} H_{23}+ \\
& +2 H^{\prime}{ }_{22} H_{01} H_{03} H_{13}+2 H^{\prime}{ }_{33} H_{01} H_{02} H_{12}+H_{01}^{2} H_{23}^{2}+H_{02}^{2} H_{13}^{2}+H_{03}^{2} H_{12}^{2}- \\
& -2 \mathrm{H}_{01} \mathrm{H}_{02} \mathrm{H}_{13} \mathrm{H}_{23}-2 \mathrm{H}_{01} \mathrm{H}_{03} \mathrm{H}_{12} \mathrm{H}_{23}-2 \mathrm{H}_{02} \mathrm{H}_{03} \mathrm{H}_{12} \mathrm{H}_{13}+ \\
& +\Delta^{2}\left[H_{01}^{2}+H_{02}^{2}+H_{03}^{2}-H^{\prime}{ }_{00}\left(H^{\prime}{ }_{11}+H^{\prime}{ }_{22}+H^{\prime}{ }_{33}+2 \Delta\right)\right]=0 \text {, }
\end{aligned}
$$

\begin{tabular}{|c|c|c|c|c|c|c|c|c|}
\hline & $E_{g}$ & $\varepsilon_{0}$ & $\varepsilon_{1}$ & A & $B$ & (ss) & $(s p)$ & $\Delta$ \\
\hline & & & & & & & & \\
\hline $\begin{array}{l}\mathrm{KCl} \\
\mathrm{KBr} \\
\mathrm{KI}\end{array}$ & $\begin{array}{l}8,65 \\
7,50 \\
6,34\end{array}$ & $\begin{array}{l}2,172 \\
2,004 \\
2,004\end{array}$ & $\begin{array}{l}-9,028 \\
-8,036 \\
-7,176\end{array}$ & $\begin{array}{l}0,073 \\
0,080 \\
0,098\end{array}$ & $\begin{array}{l}-0,050 \\
-0,057 \\
-0,064\end{array}$ & $\begin{array}{r}-0,181 \\
-0,167 \\
-0,167\end{array}$ & $\begin{array}{l}0,4806 \\
0,5150 \\
0,5640\end{array}$ & $\begin{array}{l}0,040 \\
0,170 \\
0,376\end{array}$ \\
\hline
\end{tabular}

где $H_{\alpha \alpha}^{\prime}=H_{\alpha \alpha}-E(\mathbf{k})$.

Значения $E_{g}$ и параметров для кристаллов $\mathrm{KCl}, \mathrm{KBr}$ и $\mathrm{KI}, ~ э B$

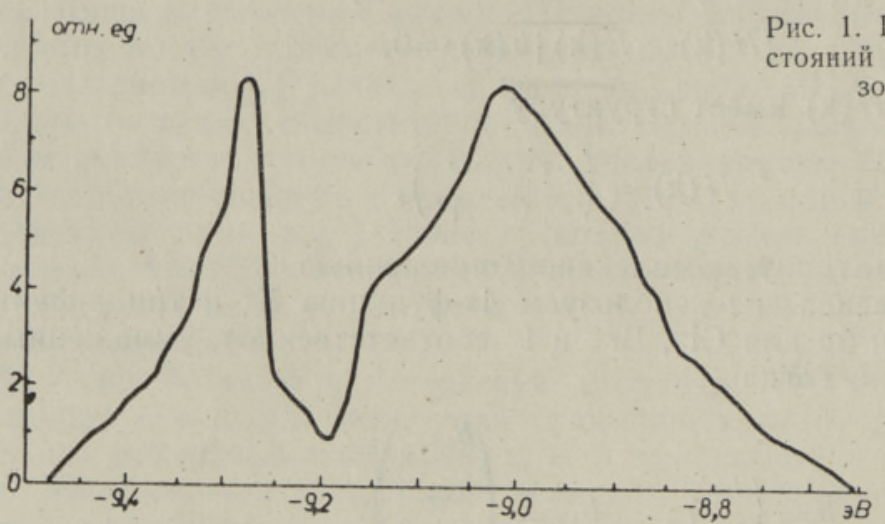

Параметры для $\mathrm{KCl}$ брались из $\left[{ }^{8}\right]$, а для $\mathrm{KBr}$ и $\mathrm{KI}$ определялись на основании экспериментального значения запрещенной щели в точке $\Gamma\left(E_{g}\right)$ и значений энергий в симметричных точках $L$ и $X$, вычисленных в $\left[{ }^{9}\right]$. Значения $E_{g}$ и всех параметров приведены в таблице (в обозна-

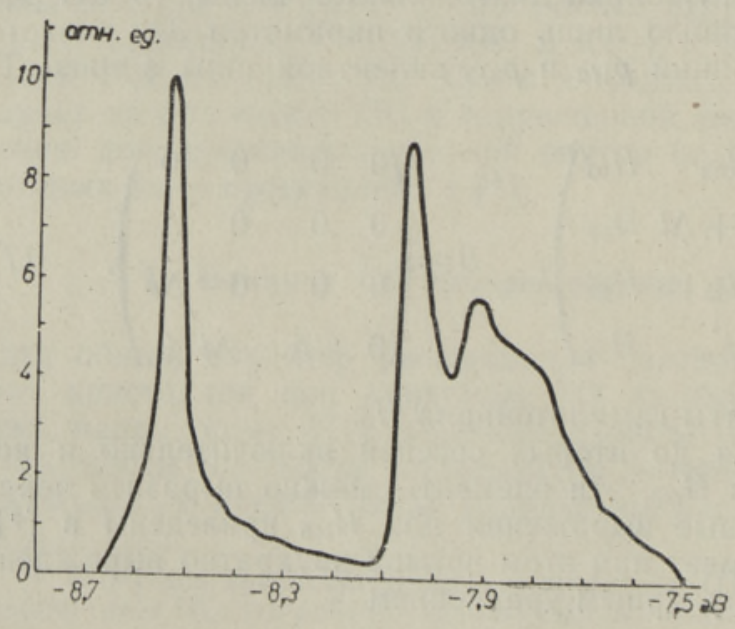

чениях $\left.\left[{ }^{8}\right]\right)$, причем за нуль энергий выбрано дно зоны проводимости.

Энергетическая структура вычислялась в 8000 точках зоны Бриллюэна и на основе этих значений энергий рассчитывались плотности состояний валентных зон (рис. $1-3$ ).

Плотность состояния валентной зоны $\mathrm{KCl}$ (рис. 1) напоминает плотность,

Рис. 2. Плотность состояний в валентной зоне $\mathrm{KBr}$. 


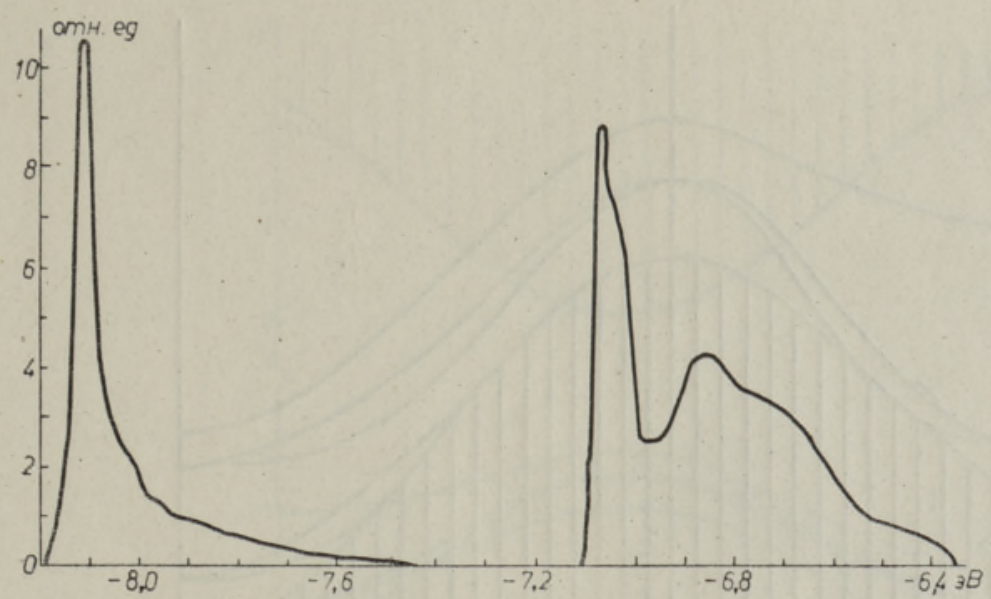

Рис. 3. Плотность состояний в валентной зоне КI.

вычисленную в $\left[{ }^{8}\right]$ (без учета СО взаимодействия), т. е. имеет также два максимума и минимум при энергии $-9,2$ э $B$, а ширина всей зоны 0,83 эB. Для $\mathrm{KBr}$ (рис. 2) имеются три максимума при энергиях $-7,92 ;-8,05 ;-8,54$ эВ. Последний пик обусловлен СО взаимодействием. Ширина зоны 1,17 эВ. Аналогичные пики образуются в КI (рис. 3) при энергиях $-6,86 ;-7,06 ;-8,11$ эВ. Между последними появляется запрещенная щель шириной 0,37 эB. Ширины подзон 0,76 эВ (верхняя) и 0,72 эB, а всей валентной зоны 1,85 эB.

\section{2. Электронная структура полуограниченного кристалла}

Поверхность моделируем, как и в $\left[{ }^{4}\right]$, «разрезанием» кристалла между плоскостями (100) и (000). Тогда уравнение Шредингера для системы из двух идентичных кристаллов с поверхностями типа (100) можно представить с помощью метода функций Грина в виде

$$
C=G W C,
$$

где $C$ - матрица волновых функций системы в узельном представлении, $G$ - матрица функций Грнна для бесконечного кристалла с учетом CO взаимодействия, $W$ - матрица возмущения, вносимого разрезанием кристалла. В последнюю матрицу входят члены, учитывающие исчезновение взаимодействия между ионами, расположенными по разные стороны от разрезающей плоскости, и изменение кулоновских интегралов $\varepsilon_{0}, \varepsilon_{1}$ для поверхностных ионов. Предполагаем, что определенное таким образом поверхностное возмущение не зависит от спина. Тогда, используя обозначения $\left[{ }^{4}\right]$ и учитывая конкретный вид матрицы $W$, получим после двумерного преобразования Фурье для компоненты волновой функции $C_{l_{1}}^{\alpha}$, перпендикулярной к поверхности, систему уравнений

$$
\begin{gathered}
C_{l_{1}^{\prime}}^{\alpha}=\sum_{\substack{v=+.-; \\
l_{1}=0,1}}\left\{g_{l_{1}^{\prime} l_{1}}^{\alpha s v}\left[\Delta \varepsilon_{0} C_{l_{1}}^{s v}-2(s s) D C_{\gamma}^{s v}-\left(\gamma-l_{1}\right)(s p) C_{\gamma}^{x v}\right]+\right. \\
+g_{l_{1}^{\prime} l_{1}}^{\alpha x v}\left[\Delta \varepsilon_{1}^{\prime} C_{l_{1}}^{x v}+\left(\gamma-l_{1}\right)(s p) C_{\gamma}^{s v}-A D C_{\gamma}^{x v}+i B\left(\gamma-l_{1}\right)\left(C_{\gamma}^{y v} \sin a k_{y}+\right.\right.
\end{gathered}
$$




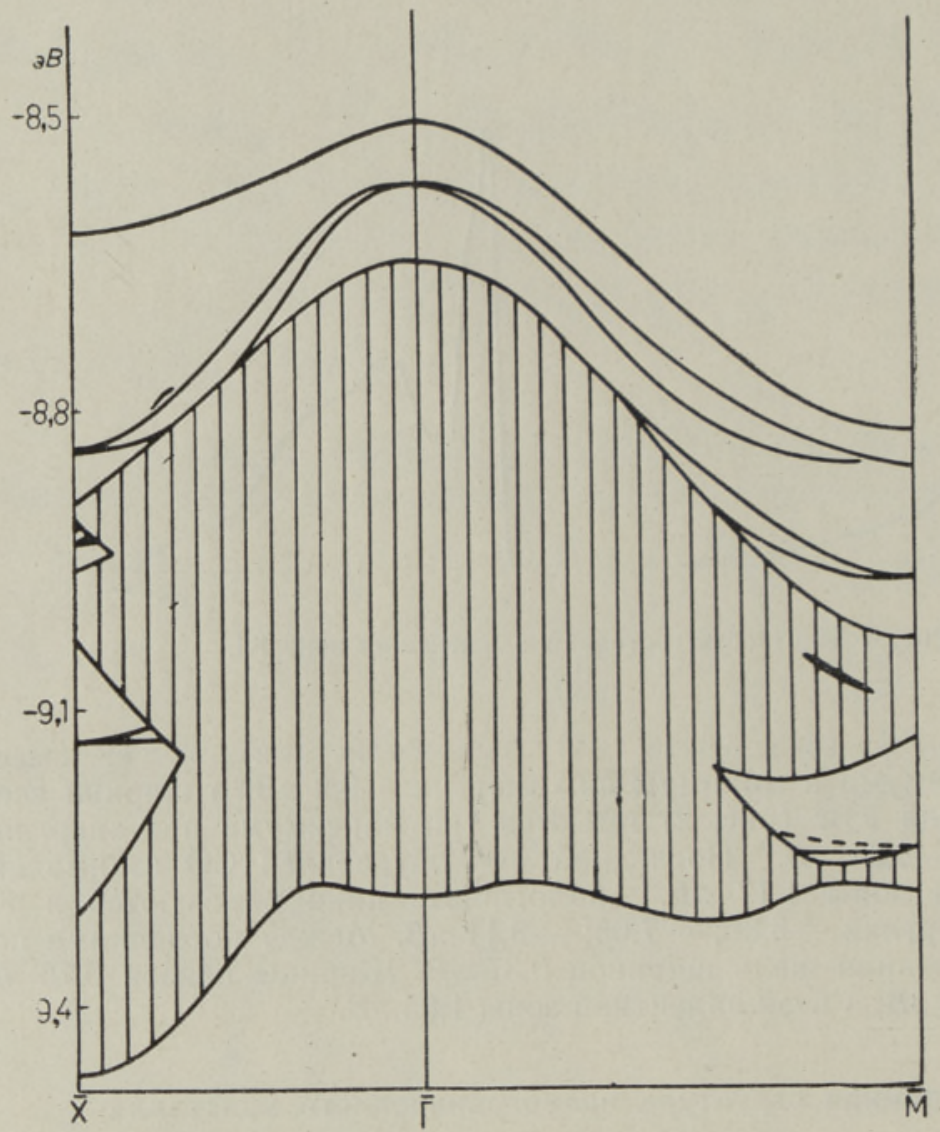

Рис. 4. Энергетические уровни в области валентной зоны

на поверхности

(100) KCl. Заштрихованные участки непрерывный спектр (здесь и на рис. 5, $6)$.

$\left.\left.+C_{\gamma}^{z v} \sin a k_{z}\right)\right]+g_{l^{\prime} l_{1}}^{\alpha v v}\left[\Delta \varepsilon_{1}^{\prime \prime} C_{l_{1}}^{y v}-\left(A D+B \cos a k_{z}\right) C_{\gamma}^{y v}+\right.$

$\left.+i B\left(\gamma-l_{1}\right) C_{\gamma}^{x v} \sin a k_{y}\right]+g_{l_{1} l_{1}}^{\alpha z v}\left[\Delta \varepsilon_{1}^{\prime \prime} C_{l_{1}}^{z v}-\left(A D+B \cos a k_{y}\right) C_{\gamma}^{z v}+\right.$

$\left.\left.+i B\left(\gamma-l_{1}\right) C_{\gamma}^{x v} \sin a k_{z}\right]\right\}$,

где $\gamma=l_{1}+(-1)^{l_{1}}, \quad D=\cos a k_{y}+\cos a k_{z}$,

$$
g_{l_{1} l_{1}^{\prime}}^{\alpha \beta}\left(E, k_{y}, k_{z}\right)=N^{-1 / 3} \sum_{n, k_{x}} \frac{b_{\alpha}(\mathrm{k}, n) b_{\beta}^{*}(\mathrm{k}, n)}{E-E_{n}(\mathrm{k})} \exp \left\{i a k_{x}\left(l_{1}-l_{1}^{\prime}\right)\right\} .
$$

Индексы $l_{1}$ и $l_{1}^{\prime}$ принимают значения 0 и $1 ; \alpha-s v^{\prime}, x v^{\prime}, y v^{\prime}, z v^{\prime}$ $\left(v^{\prime}=+,-\right)$.

Решение системы (10) определяет энергии и волновые функции ПС. Учитывая симметрию, можно понизить ранг определителя этой системы до восьми.

Энергии ПС в кристаллах $\mathrm{KCl}, \mathrm{KBr}, \mathrm{KI}$ для направлений $\vec{\Gamma} \vec{M}$ и $\bar{\Gamma} \bar{X}$ рассчитывались нами в двумерной зоне Бриллюэна, соответствующей поверхности (100). Функции Грина $g_{l}^{\alpha \beta},{ }_{11}^{\alpha}$ вычислялись в рамках модели, описанной в п. 1. Значения для изменения кулоновских интегралов $\Delta \varepsilon_{0}, \Delta \varepsilon_{1}{ }^{\prime}, \Delta \varepsilon_{1}{ }^{\prime \prime}$ поверхностных ионов для $\mathrm{KCl}$ брались из [ [ ${ }^{4}$, а для $\mathrm{KBr}$ и КІ рассчитывались в предположении, что изменение короткодействующей поправки $\Delta \sigma$ для всех гантелей волновой функции равно 
Рис. 5. Энергетические уровни в области валентной зоны и зоны проводимости на поверхности (100) $\mathrm{KBr}$.

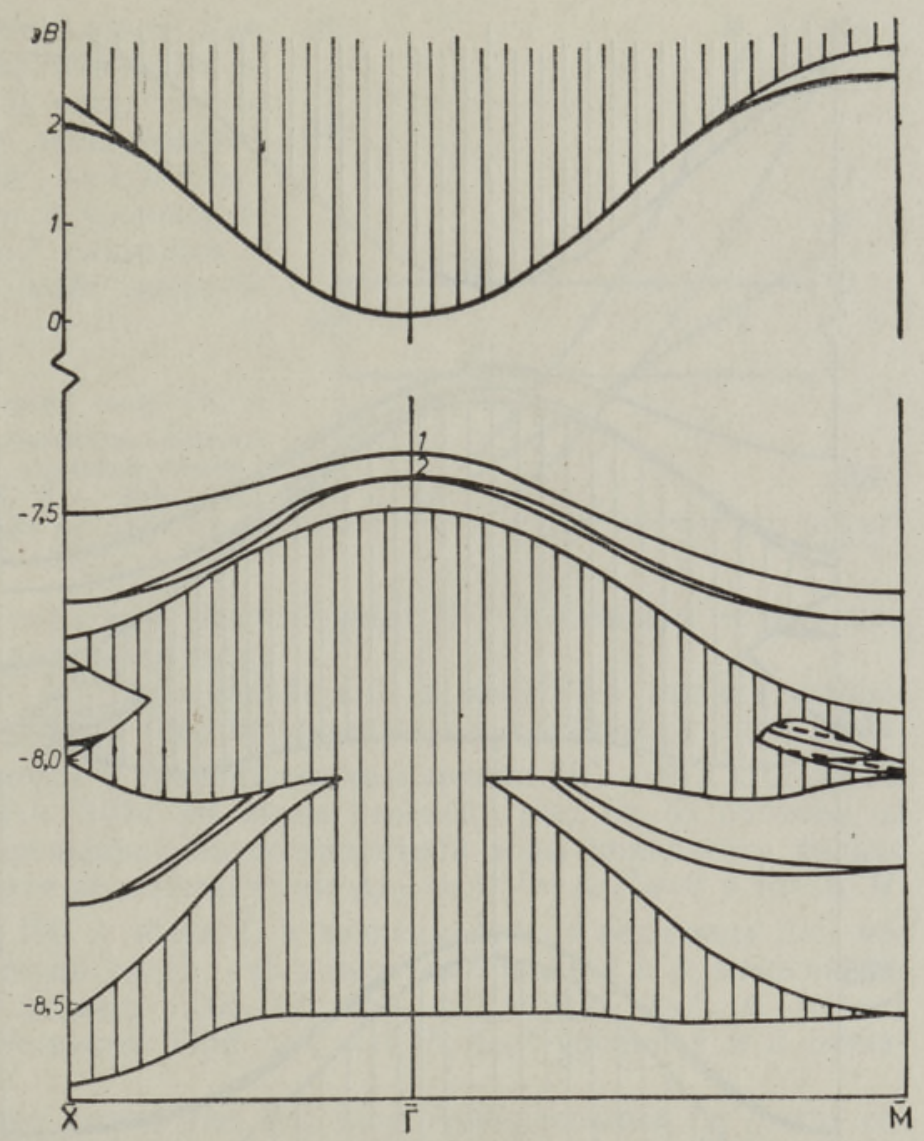

нулю. Тогда $\Delta \varepsilon_{1}^{\prime}=\Delta \varepsilon_{1}^{\prime \prime}=-\Delta \varepsilon_{0}=0,290 \quad{ }^{\prime \prime} B \quad$ для $\mathrm{KBr} \quad$ и $\quad \Delta \varepsilon_{1}^{\prime}=$ $=\Delta \varepsilon_{1}{ }^{\prime \prime}=-\Delta \varepsilon_{0}=0,273$ э $B$ для КІ. Энергии ПС и проекции объемной энергетической структуры на двумерную зону показаны на рис. 4-6. Для всех кристаллов характерно появление ПС между валентной зоной и зоной проводимости, а также в области валентной зоны в щелях запрещенной энергии. Их возникновение обусловлено образованием валентной зоны из трех энергетических ветвей [ $\left.{ }^{6}\right]$, которые в нашем случае двукратно вырождены. Поэтому при данных $k_{y}, k_{z}$ возможно появление не более двух запрещенных щелей в двумерной зоне. Нижняя щель шире из-за СО взаимодействия.

\section{3. Результаты расчетов}

KCl. Энергетическую структуру ПС у края валентной зоны (рис. 4) полезно сравнить со структурой, полученной без учета СО взаимодействия $\left[{ }^{6}\right]$. Под влиянием СО взаимодействия, кроме энергетического уровня на расстоянии 0,143 эB от зоны в точке $\bar{\Gamma}$, появляется дополнительный уровень с энергией 0,081 э $B$, который в менее симметричных точках расщепляется. Максимальное число ветвей ПС выше валентной зоны равно шести. Из-за СО взаимодействия запрещенные участки энергий внутри валентной зоны становятся шире, охватывая и бо́льшую область двумерной зоны, благодаря чему возрастает вероятность образования в них дополнительных ветвей ПС. Кроме таммовских ПС, в них 


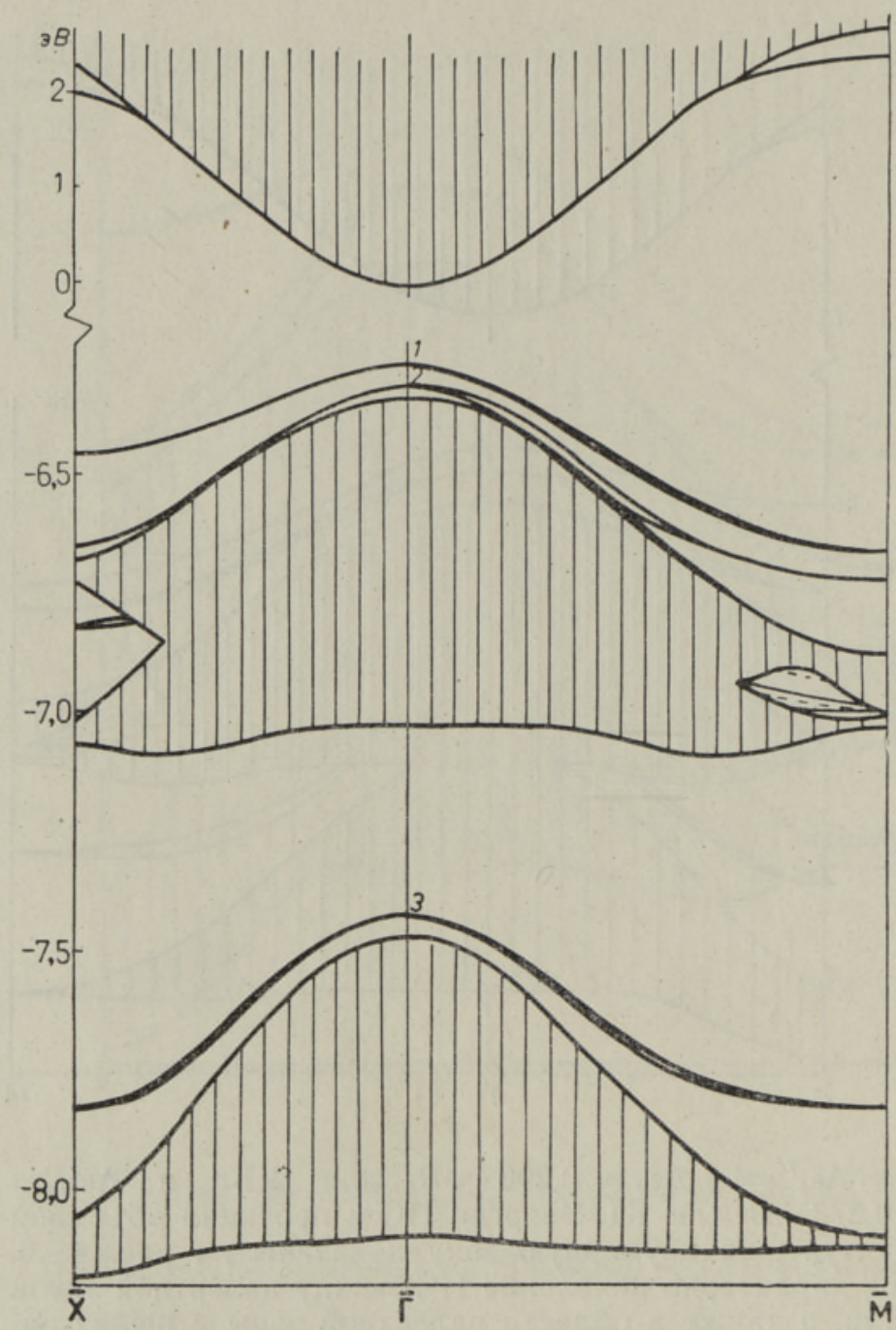

Рис. 6. Энергетические уровни в области валентной зоны и зоны проводимости на поверхности (100) KI.

появляются состояния типа Шокли. Энергии последних при $\Delta \varepsilon_{0}=$ $=\Delta \varepsilon_{1}^{\prime}=\Delta \varepsilon_{1}{ }^{\prime \prime}=0$ показаны пунктиром на рис. 4. Проекции энергий зоны проводимости и энергетическая структура ПС вблизи них совпадают с вычисленными в [ [ $\left.{ }^{4}\right]$.

$\mathrm{KBr}$. В запрещенной щели между валентной зоной и зоной проводимости появляются ПС только у валентной зоны, отщепляясь от нее в точке $\bar{\Gamma}$ на 0,120 и 0,068 э $B$ (рис. 5 ). Последние состояния двукратно вырождены в $\bar{\Gamma}$, но расщеплены в других точках на четыре ветви ПС выше валентной зоны. В верхней запрещенной щели внутри валентной зоны возможно появление двух пар ПС (у точки $\bar{X})$, а в нижней только одной пары. Если поверхностное возмущение (изменение кулоновских интегралов на поверхности) достаточно мало, то остаются два уровня (ПС типа Шокли) в верхней щели у точки $\bar{M}$. При $\Delta \varepsilon_{0}=$ $=\Delta \varepsilon_{1}^{\prime}=\Delta \varepsilon_{1}^{\prime \prime}=0$ энергия последних показана пунктиром на рис. 5 .

Нами рассчитана зависимость энергии связи ПС в точке $\bar{\Gamma}$ от величины изменения кулоновского интеграла $\Delta \varepsilon_{1}^{\prime}$ для $p$-гантелей, перпендикулярных к поверхности. В данном приближении это означает изменение значения короткодействующей поправки $\sigma$ в кулоновском интег- 
рале для $p$-состояний галоида. Как видно из рис. 7 , верхний уровень (кривая 1 на рис. 5) остается постоянным, меняется лишь нижний (кривая 2). При $\Delta \varepsilon_{1}^{\prime}=1,38 \Delta \varepsilon_{1}^{\prime \prime}$ последний совпадает с уровнем 1 , а при $\Delta \varepsilon_{1}^{\prime}<0,4 \Delta \varepsilon_{1}{ }^{\prime \prime}$ сливается с валентной зоной (имеет энергию $\left.<0,01{ }_{\ni} B\right)$.

Рис. 7. Зависимость энергии связи ПС в точке $\bar{\Gamma}$ от величины изменения кулоновского интеграла для $p_{x}$-гантелей поверхностного иона. $A-\mathrm{KI}, B-\mathrm{KBr}$, цифры соответствуют кривым на рис. 5 и 6 .

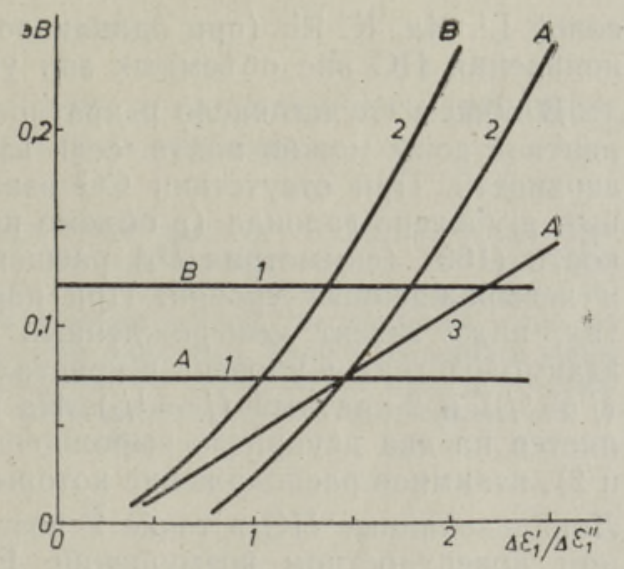

ПС у зоны проводимости (электронные ПС) возникают только при больших значениях квазиимпульса.

KI. Из-за сильного СО взаимодействия в ионе $\mathrm{I}^{-}$нижняя запрещенная щель в валентной зоне образуется и в трехмерной зоне Бриллюэна. Поэтому в точке $\bar{\Gamma}$ появляются три уровня ПС: 0,073 и 0,024 эВ выше верхней зоны и 0,044 э $B$ выше нижней (рис. 6). В отличие от $\mathrm{KBr}$, в верхней запрещенной щели валентной зоны появляется только одна пара ПС, которая не исчезает при $\Delta \varepsilon_{0}=\Delta \varepsilon_{1}^{\prime}=\Delta \varepsilon_{1}^{\prime \prime}=0$ у точки $\bar{M}$. Изменение энергий ПС в точке $\bar{\Gamma}$ в зависимости от величины $\Delta \varepsilon_{1}^{\prime}$ показано на рис. 7 , откуда видно, что меняются уровни 2 и 3 , энергии которых становятся меньше 0,005 эB при $\Delta \varepsilon_{1}^{\prime}<0,75 \Delta \varepsilon_{1}^{\prime \prime}$ и $\Delta \varepsilon_{1}^{\prime}<$ $<0,30 \Delta \varepsilon_{1}^{\prime \prime}$ соответственно. При $\Delta \varepsilon_{1}^{\prime}=1,45 \Delta \varepsilon_{1}^{\prime \prime}$ уровни 1 и 2 совпадают.

Нами исследовано появление ПС при отрицательных $\Delta \varepsilon^{\prime}{ }_{1}$, что соответствует условию $\sigma>2 \alpha$ ( $\alpha$ - изменение энергии Маделунга). Ниже верхней валентной зоны возникает локальный уровень при $\Delta \varepsilon_{1}^{\prime}<$ $<-\Delta \varepsilon_{1}^{\prime \prime}$ (в точке $\left.\bar{M}\right)$, который, однако, попадает в запрещенную щель трехмерной зоны Бриллюэна лишь при $\Delta \varepsilon_{1}^{\prime}<-1,5 \Delta \varepsilon_{1}{ }^{\prime \prime}$. При последнем условии появляются уровни и ниже второй валентной зоны. Однако необходимые для отрицательных значений $\Delta \varepsilon_{1}^{\prime}$ большие величины $\sigma$ вряд ли могут реализоваться.

ПС у зоны проводимости существуют (как и в $\mathrm{KCl}, \mathrm{KBr}$ ) только у граней двумерной зоны Бриллюэна. В запрещенной щели (у дна зоны проводимости в точке $\bar{\Gamma}$ ) они появляются (энергия их становится больше 0,01 э $B$ ) при $\Delta \varepsilon_{0}<-0,84$ ә $; ; \Delta \varepsilon_{0}<-0,79$ э $B ; \Delta \varepsilon_{0}<-0,78$ э $B$ для $\mathrm{KCl}$, $\mathrm{KBr}$, KI соответственно.

\section{4. Обсуждение}

На основании проведенных расчетов можно сделать выводы о существовании и расположении энергетических уровней ПС в других щелочногалоидных кристаллах со структурой $\mathrm{NaCl}$. Поскольку валентные зоны этих кристаллов образованы в основном из p-состояний иона галоида, то и ПС, отщепляющиеся от этих зон, зависят от типа иона галоида. Поэтому можно думать, что во всех хлоридах, бромидах и иодидах для энергетической структуры на поверхности (100) получатся картины, аналогичные приведенным выше. Как легко показать, изменение энергии Маделунга для поверхностных ионов, которое дает основной вклад в члены поверхностного возмущения $\Delta \varepsilon_{0}, \Delta \varepsilon^{\prime}{ }_{1}, \Delta \varepsilon_{1}{ }^{\prime \prime}$, уменьшается в ряду 
солей $\mathrm{Li}, \mathrm{Na}, \mathrm{K}, \mathrm{Rb}$ (при одинаковом анионе). Поэтому и возможность появления ПС вне объемных зон уменьшается в этих рядах.

В общем случае число и кратности вырождения ПС в точке $\bar{\Gamma}$ у валентной зоны можно найти, если известна симметрия кристалла на поверхности. При отсутствии СО взаимодействия трехкратно вырожденный $p$-уровень галоида (в объеме кристалла симметрия $O_{h}$ ) на поверхности (100) (симметрия $D_{4}$ ) расщепляется на двукратно вырожденный и невырожденный уровни. При параметрах, использованных в расчетах, ниже лежит невырожденный уровень $\left[{ }^{4,6}\right]$. Под влиянием СО взаимодействия $p$-уровень в кристалле расщепляется на два: 4-кратный $(j=3 / 2)$ и 2 -кратный $(j=1 / 2)$. На поверхности первый из них расщепляется на два двукратно вырожденных уровня (на рис. 5 и 6 кривые 1 и 2), взаимное расположение которых зависит от величины изменения $\sigma$. Локализованные ПС в точке $\bar{\Gamma}$ возникают только при достаточно сильном поверхностном возмущении. Если последнее для этого недостаточно, то ПС (или некоторые из них) переходят в псевдолокальные ПС (поверхностные резонансы).

Появление запрещенных щелей в валентной зоне в двумерной зоне Бриллюэна определяется деталями зонной структуры, а вероятность возникновения их увеличивается в рядах $\mathrm{F}, \mathrm{Cl}, \mathrm{Br}$, I из-за СO взаимодействия. Возможность появления в этих щелях ПС зависит от ширины щели и величины поверхностного возмущения. Влияние последнего, однако, невелико и тоже зависит от ширины щели.

\section{Л И ТЕРАТ У Р А}

1. Saile, V., Skibovski, M., Steinmann, W., Gürtler, P., Koch, E., Ko z e vn ikov, A., Phys. Rev. Lett., 37, 305 (1976).

2. Henrich, V. E., Dresselhaus, G., Z eiger, H. J., Phys. Rev. Lett., 36, 158 (1976).

3. Levine, J. D., Mark, P., Phys. Rev., 144, 751 (1966).

4. 3 а в т Г. С., С а к С Т. Я., ФТТ, 14, 2897 (1972).

5. Bertoni, C. M., Calandra, C., Phys. Stat. Sol., B50, 527 (1972).

6. С а к с Т. Я., 3 а в т Г. С., ФТТ, 19, 1856 (1977).

7. K nox, R., T e e g a rde n, K., In: Physics of Color Centers, ed. W. B. Fowler, New York, 1968, p. 1.

8. Шуличенко Б. В., З авт Г. С., Кристофель Н. Н., ФТТ, 12, 2621, (1970); Препринт ИФА-4, Тарту, 1970.

9. Ku nz, A. B., J. Phys. Chem. Solids, 31, 265 (1970); Over hof, H., Phys. Stat. Sol, B43, 575 (1971).

Институт физики
Академии наук Әстонской ССР

Поступила в редакцию $8 / \mathrm{IV} 1977$

\section{IOONKRISTALLIDE PINNA (100) ELEKTRONOLEKUD} SPINNORBITAALSE INTERAKTSIOONI KORRAL

On esitatud $\mathrm{KCl}, \mathrm{KBr}$ ja $\mathrm{KI}$ kristallide valentstsooni ja juhtivustsooni alumise osa elektronstruktuuri arvutus, kusjuures on arvestatud spinnorbitaalset interaktsiooni valentstsoonis ja kasutatud Slater-Kosteri interpolatsiooniskeemi LCAO lähenduses. Greeni funktsioonide meetodil on arvutatud samade kristallide elektronolekud pinnal (100) ning leitud, et pinnaolekud tekivad nii valents- ja juhtivustsooni vahel kui ka spinnorbitaalsest interaktsioonist tingitud keelupilus ja valentstsooni pideva spektri piirkonnas. Osa viimaseid pinnaolekuid kuulub Shockley tüüpi olekute hulka. 


\section{ELECTRONIC STATES AT (100) SURFACE OF IONIC CRYSTALS WITH SPIN-ORBIT INTERACTION TAKEN INTO ACCOUNT}

The electronic structure of valence bands and lower parts of conduction bands have been calculated for the crystals $\mathrm{KCl}, \mathrm{KBr}, \mathrm{KI}$, taking into account the spin-orbit (SO) interaction. The Slater-Koster interpolation procedure has been used in the LCAO method. For these crystals, using the Green function method, the electronic states at the (100) surface have been calculated. It has been found that, besides the surface states (SS) between the valence and conduction band, new SS appear in the band gap caused by the SO interaction, and also inside the continuous region of the valence band. A part of the latter SS belong to the Shockley-type states. 